\title{
A comparison between FUV remote sensing of magnetotail stretching and the T01 model during quiet conditions and growth phases
}

\author{
C. Blockx ${ }^{1}$, J.-C. Gérard ${ }^{1}$, V. Coumans ${ }^{1}$, B. Hubert ${ }^{1}$, and M. Meurant ${ }^{2}$ \\ ${ }^{1}$ Laboratoire de Physique Atmosphérique et Planétaire, Université de Liège, Liège, Belgium \\ ${ }^{2}$ Institute for Space Research - University of Calgary, Calgary, Canada
}

Received: 30 June 2006 - Revised: 21 November 2006 - Accepted: 29 November 2006 - Published: 1 February 2007

\begin{abstract}
In a previous study, Blockx et al. (2005) showed that the SI12 camera on board the IMAGE spacecraft is an excellent tool to remotely determine the position of the isotropy boundary (IB) in the ionosphere, and thus is able to provide a reasonable estimate of the amount of stretching of the magnetic field lines in the magetotail. By combining an empirical model of the magnetospheric configuration with Sergeev's criterion for non-adiabatic motion, it is also possible to obtain a theoretical position of IB in the ionosphere, for known conditions in the solar wind. Earlier studies have demonstrated the inadequacy of the Tsyganenko-1989 (T89) model to quantitatively reproduce the field line stretching, particularly during growth phases. In this study, we reexamine this question using the T01 model which considers the time history of the solar wind parameters. We compare the latitude of IB derived from SI12 global images near local midnight with that calculated from the T01 model and the Sergeev's criterion. Observational and theoretical results are found to frequently disagree. We use in situ measurements of the magnetic field with the GOES-8 satellite to discriminate which of the two components in the calculation of the theoretical position of the IB (the T01 model or Sergeev's criterion) induces the discrepancy. For very quiet magnetic conditions, we find that statistically the T01 model approximately predicts the correct location of the maximum proton precipitation. However, large discrepancies are observed in individual cases, as demonstrated by the large scatter of predicted latitudes. For larger values of the AE index, the model fails to predict the observed latitude of the maximum proton intensity, as a consequence of the lack of consideration of the cross-tail current component which produces a more elongated field configuration at the location of the proton injection along the field lines. We show that it is possible to match the observed location of the maximum proton precip-
\end{abstract}

Correspondence to: C. Blockx

(c.blockx@ulg.ac.be) itation by decreasing the current sheet half-thickness $D$ parameter. We thus conclude that underestimation of the field line stretching leads to inadequately prediction of the boundary latitude of the non-adiabatic proton precipitation region.

Keywords. Magnetospheric physics (Auroral phenomena; Magnetotail; Solar wind-magnetosphere interactions)

\section{Introduction}

The isotropy boundary (IB) introduced by Sergeev et al. (1983) is the low-altitude signature of the transition from the region of the magnetosphere dominated by strong pitch angle scattering to the region of bounce-trapped particles. This transition also marks the equatorward boundary of significant ion precipitation. Equatorward of this boundary, the ion distributions on closed field lines may display a strong flux depletion in the loss cone due to collisions in the ionosphere. In the absence of wave-particle interaction, the depleted loss cone will be conserved for adiabatically moving particle. However, possible nonadiabaticity or stochastization of particle motion will lead to the filling of the loss cone. In particular, when observing the downgoing particles at low altitude above the ionospheric loss region, the relative amount of particles inside the loss cone can be used to measure the amount of pitch angle scattering during one bounce between the opposite mirror points. For those particles that mirror at low altitudes (having small equatorial pitch angles), the deviations from adiabatic motion are strongest at the equator in the central current sheet. Adiabaticity is primarily controlled by the equatorial value of the ratio $R=R c / \rho$, where $R c$ is the curvature radius of the field line, and $\rho$ is the particle gyroradius. According to numerical simulations of trajectories of small pitch angle particles, the threshold condition for strong pitch angle scattering (scattering to the

Published by Copernicus GmbH on behalf of the European Geosciences Union. 
center of loss cone) is approximately as follows (Sergeev et al., 1993):

$R=R c / \rho \leq 8$.

Assuming that the magnetic field decreases monotonically tailward, the isotropy boundary is located where the equality sign holds. Since the location of the IB is controlled by the magnetic field in the current sheet, the IB position reflects changes in the equatorial magnetic field in the near tail and can be used to monitor these changes. The latitude of this boundary depends on the energy of the particles (Sergeev et al., 1983), with the lower latitudes associated with the highest energies.

The IB is well identified from in situ measurements with low-altitude polar spacecraft such as NOAA and FAST satellites. When these spacecraft cross the auroral oval, they provide the flux inside and outside the loss cone. Direct determination of this boundary is not possible from the Defense Meteorological Satellite Program (DMSP) satellites because of the lack of pitch angle coverage (only precipitation fluxes are measured). However, Newell et al. (1998) showed a close association between the IB of $30 \mathrm{keV}$ protons detected with NOAA satellites and the $b 2 i$ boundary defined as the latitude of the ion energy flux precipitation maximum of 3-30 keV protons observed with the DMSP satellites. The knowledge of the latitude of this boundary in the nightside proton precipitation provides considerable information on the state of the magnetosphere, as it is an indicator of the level of magnetic field line stretching in the magnetotail. Actually, Sergeev and Gvozdevsky (1995) showed that unlike geomagnetic indices such as $K_{p}$, the magnetotail (MT) index, defined as the invariant latitude of the isotropy boundary of $\sim 100 \mathrm{keV}$ protons reduced to the midnight meridian, displays a good correlation $(r \sim 0.9)$ with the inclination of the magnetic field measured in the nightside portion of a geosynchronous orbit.

Ground-based remote sensing of the IB location was described by Donovan et al. (2003) and Nicholson et al. (2002). Donovan et al. (2003) used ion data from DMSP overflights of the Canadian Auroral Network for the OPEN Program Unified Study (CANOPUS) meridian scanning photometer (MSP) located at Gillam, Canada, to develop a simple algorithm to identify the "optical $b 2 i$ boundary" in latitude profiles of proton auroral $\mathrm{H}_{\beta}(486 \mathrm{~nm})$ brightness. The latitude of this optical $b 2 i$ is

$b 2 i=\Lambda_{\text {peak }}-1.4 \sigma$

where $\Lambda_{\text {peak }}$ and $\sigma$ are the latitude of the peak and the standard deviation of the Gaussian fit to the brightness profile, respectively. They found by intercomparison of $\sim 1600$ nearsimultaneous optical and in situ $b 2 i$ that the optical $b 2 i$ is a reasonable basis for an optical equivalent to the MT index put forward by Sergeev and Gvozdevsky (1995). They also demonstrated a strong correlation between the optical $b 2 i$ and the inclination of the magnetic field as measured at GOES 8 and developed an empirical model for predicting the GOES 8 inclination. Nicholson et al. (2002) used data obtained from MSPs located at Gillam and Fort Smith, Canada, and Poker Flat, Alaska, and demonstrated that ground-based MSPs are capable of obtaining a large number of simultaneous boundary determinations.

\section{The SI12 proton imager}

Satellite-based observations of the proton aurora (and thus of the proton IB) were so far restricted to in situ satellite particle detection. Until the availability of the IMAGE satellite, no capability existed for global remote sensing of the proton aurora. Consequently, the IB sampling was spatially and temporally restricted. To overcome this limitation, Blockx et al. (2005) proposed to use global data obtained with the FUV spectrographic proton imager (SI12) on board IMAGE that provide snapshots of proton precipitation with a 2-min resolution. Since the launch of the IMAGE satellite in March 2000 until December 2005, the FUV instrument provided the capability to simultaneously observe the aurora in three spectral bands:

- The Wideband Imaging Camera (WIC) observes the aurora in a broad $(135-170 \mathrm{~nm})$ ultraviolet band sensitive mainly to Lyman-Birge-Hopfield $\mathrm{N}_{2}$ bands and NI lines.

- The SI12 spectral imager isolates a narrow region $(0.2 \mathrm{~nm})$ with a peak sensitivity at $121.8 \mathrm{~nm}$ and images Doppler shifted Ly- $\alpha$ auroral emission.

- The SI13 spectral imager selects a $5 \mathrm{~nm}$ passband centered on the O I $135.6 \mathrm{~nm}$ feature.

The response of these instruments was validated by laboratory and in-flight calibrations using hot stars and was updated daily (Frey et al., 2003).

SI12 was designed to monitor the global-scale proton precipitation. It includes a grill system to reject the intense $(>10 \mathrm{kR})$ geocoronal Ly- $\alpha$ emission at $121.5 \mathrm{~nm}$, which would otherwise appear as an bright diffuse glow, and to isolate a fraction of the Doppler-broadened auroral Ly- $\alpha$ line profile (Mende et al., 2000a, b). Since this emission is emitted by excited fast hydrogen atoms, the line profile for a given observation geometry depends on the energy spectrum of the incident auroral protons and their pitch angle distribution. In addition, the response of the SI12 instrument with its multiple passbands depends on the Ly-a line profile (Gérard et al., 2001). The shape of this profile is also influenced by the orientation of the line of sight with respect to the local magnetic field lines. The complexity of the atmospheric and instrumental processes involved makes it necessary to simulate the SI12 response for a range of parameters describing the proton precipitation and the geometry of the observations. The drop in efficiency of the SI12 detector for energy higher than $\sim 10 \mathrm{keV}$ results from the increasing importance of other 
processes such as ionization competing with excitation into the $\mathrm{H}(2 \mathrm{p})$ state as the initial proton energy increases (Strickland et al., 1993; Gérard et al., 2000). The emission rates are calculated for a nadir-viewing observation, assuming that the pixels are uniformly filled by the Ly- $\alpha$ auroral emission. The instrument efficiency also drops for low energy proton precipitation owing to the lack of an extended red-shifted Ly- $\alpha$ wing. This rapidly varying sensitivity at low energies also limits the accuracy of the signal conversion into energy flux units. The proton energy fluxes are then obtained from the SI12 count rate using the calibrated relationship between the SI12 signal and the NOAA in situ measurements of proton precipitation (Coumans et al., 2002). This method was validated by comparisons with in situ measurements of the auroral particle energy flux obtained from FAST (Frey et al., 2001; Gérard et al., 2001; Bisikalo et al., 2003), NOAA (Hubert et al., 2002; Coumans et al., 2002; Meurant et al., 2003), and DMSP (Coumans et al., 2004) satellites.

In a previous paper, Blockx et al. (2005) showed the latitude of the isotropy boundary may be determined using SI12 global data from the latitude of the maximum proton precipitation observed by this instrument. For this purpose they compared the location of the IB determined from in situ particle measurements with that of the maximum proton precipitation observed with SI12. Parameters such as the magnetic local time and the magnetic activity index AE exert control on this location. This first result coupled with the study of Sergeev and Gvozdevsky (1995) led to the suggestion that a direct relation exists between MAX SI12 and the magnetic elevation angle. Indeed, Blockx et al. (2005) also showed that MAX SI12, as well as the IB, is a good indicator of the stretching of the magnetic field lines. They found the same level of correlation $(r \sim 0.9)$ and a similar standard deviation ( $s=0.89$ ) as was obtained by Sergeev and Gvozdevsky (1995) on the basis of in situ measurements. Both the IB and MAXSI12, unlike the usual global activity indices (magnetic indices like $\mathrm{AE}, K_{p}, D_{s t}$ ), are well suited to predict the instantaneous magnetospheric configuration in the midnight sector. Since the IMAGE satellite performs global remote sensing of the proton aurora over a long continuous time interval, and since the latitude of MAX SI12 is closely related to the IB, Blockx et al. (2005) concluded it is possible to follow the dynamic evolution of the magnetic field lines on a global scale. They showed brief and localized magnetic field perturbations (even observed at 18:00 MLT, where GOES 8 was not at the most favorable position) which evolve self-consistently with the proton auroral morphology and brightness and were also observed with the SI12 imager. The response of these perturbations detected by SI1 2 occurred simultaneously with the magnetic field disturbances.

\section{Empirical models}

The Tsyganenko-2001 model (T01) is an empirical magnetic field model representing the variable configuration of the inner and near magnetosphere for different interplanetary conditions and ground disturbance level. T01 is the evolution of successive efforts (Tsyganenko and Usmanov, 1982; Tsyganenko, 1987, 1989, 1995), resulting in progressively more refined models (T89, T96), useful in many studies. The general approach remained the same as in the earlier models (a data-based approach), but the mathematical description of all major sources of the magnetospheric field was based on a recently developed new method. In particular, T01 is the first magnetospheric model in which the partial ring current and the dawn-dusk asymmetry were explicitly taken into account (Tsyganenko et al., 2002).

Pulkkinen (1991) studied the magnetic field and electric current distributions in the magnetotail during the growth phase of substorms, using Tsyganenko's T89 magnetic field model. She constructed a temporally evolving magnetic field model for the growth phase by enhancing the near-Earth currents and thinning the current sheet from the values given by the static T89 model. In her model field, both the thinning of the current sheet and the increase in the tail flux stretch field lines tailward, thus making the field more tail-like even in the near-Earth region. She showed that if a field-aligned mapping is performed from the ionosphere into the magnetotail, the endpoint at the current sheet using the modified model can vary by more than $10 R_{E}$ from the result obtained with the basic T89 model. The ionospheric footprint of the tail-like field lines moved several degrees equatorward. Inversely, the mapping into the equatorial plane using the unmodified T89 model of an auroral arc recorded by all-sky cameras moved earthward by several $R_{E}$ during the growth phase of a substorm. This was interpreted as the signature of the inadequacy of the T89 model to reproduce the field line stretching occurring during the growth phase: the earthward motion of the mapping can be replaced by field line stretching, so that the mapping of the arc stays at a constant distance from the Earth, to correspond to the assumed development during the growth phase of the arc. Moreover, if the equatorward motion of the growth phase arc is interpreted as entirely due to changes in the tail during the growth phase, this gives an estimate for the parameters in the modified model. Kubyshkina et al. (1999) introduced and tested a new approach suitable to model the magnetotail configuration during individual events. They showed, using multiple energies to deduce the isotropic boundaries, that the $B_{z}$ component in the tail typically decreases much faster (over a shorter radial distance) in observations than in the T89 model.

The objective of this study is first to use the SI12 camera to compare the morphology of the auroral proton precipitation at all local time during quiet periods with the structure of the magnetic field provided by the Tsyganenko-2001 (T01) magnetospheric model. Secondly, the SI12 camera is 


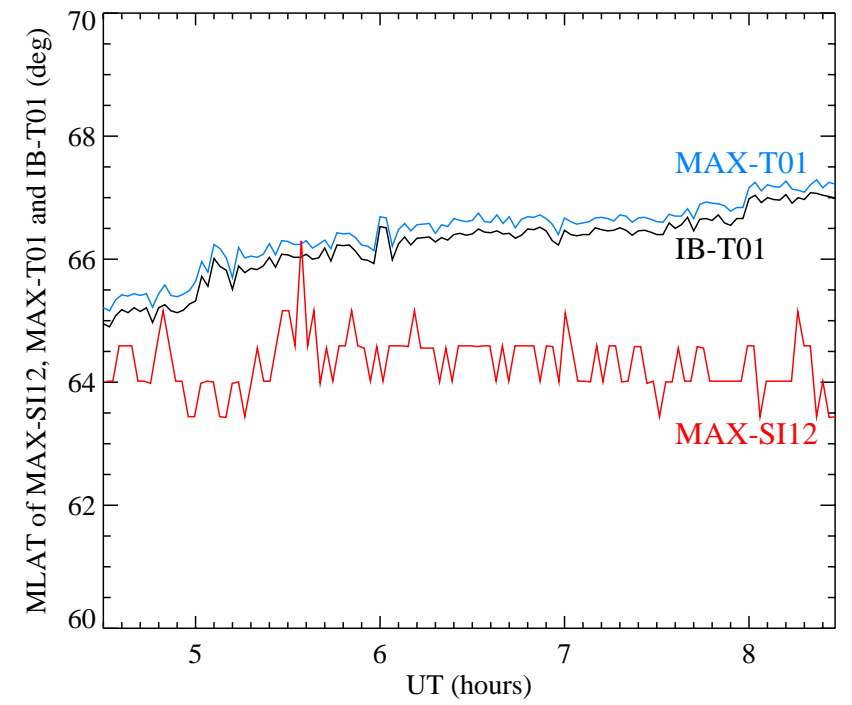

Fig. 1. Evolution of the magnetic latitude of the SI12 maximum proton precipitation (red line) during a 4-h quiet period on 5 November 2000, compared with the location of the IB $(R=8)$ (black line) and the maximum intensity $(R=6)$ (blue line) calculated by the T01 model.

used to monitor the motions of the proton precipitation during growth phases at all MLT. The growth phases are periods where the magnetic field moves slowly but with a large amplitude, and we compare the changes of latitude of the magnetic footprint with SI12 observations during such events.

\section{Results}

We first focus on the comparison between the configuration of the magnetic field (position of IB and the $R$ value) from the T01 field line model, and the SI12 observations, during quiet conditions. For this purpose, we combine the T01 model with the GEOPACK-2003 library, which includes the subroutines for the current (IGRF) internal geomagnetic field models. We use 64-s average ACE data (16-s for the magnetic field components) delayed to account for propagation to the magnetopause, corresponding to the actual values of the solar wind parameters in the example plots shown hereafter. We both examine case studies and make a statistical approach for this comparison.

For case studies, we compared MAX-SI12 for each SI12 image in the nightside sector with the calculated isotropic boundary: when moving away from the Earth in the nightside sector, we look for the T01 field lines for which the condition $R=8$ is satisfied (down-up approach). We then find the ionospheric projections (mlat, MLT) of these field lines, the calculated isotropic boundary in the ionosphere, and we compare it to the MAX-SI12. In the statistical approach, the database includes 17874 SI12 images collected in December 2000 and January 2001. For each SI12 image, we trace the

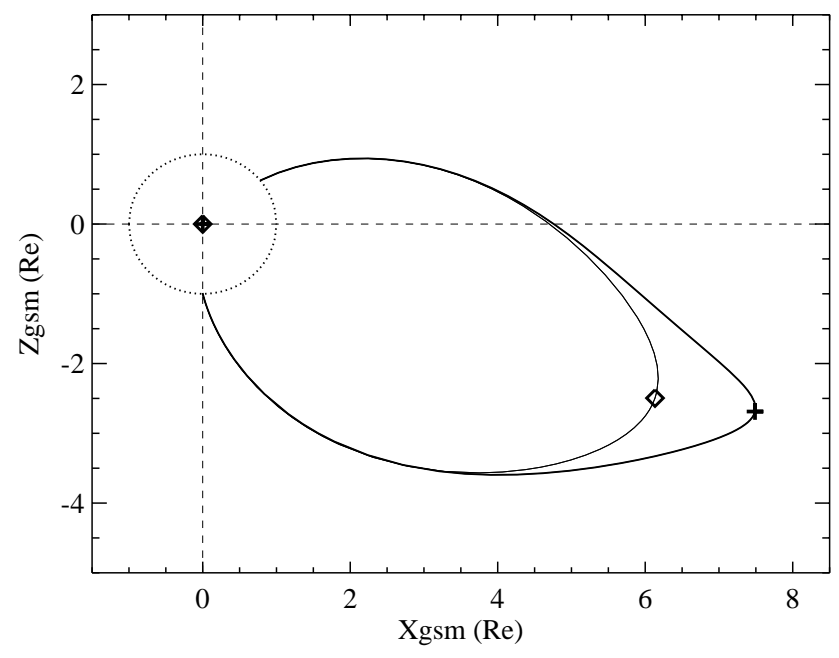

Fig. 2. Configuration of the T01 magnetic field lines whose footprints coincide with the latitude of MAX-SI12, for the actual solar wind conditions (thin solid line) and for the adjusted $D_{0}$ parameter (thick solid line) at 06:30 UT on 5 November 2000. The plus and diamond symbols indicate the apex of the magnetic field lines where $R$ corresponds to 6 and 40.2, respectively.

T01 field lines from the latitude of MAX-SI12 in the nightside sector, and calculate the $R$ value, the curvature radius $R_{C}$, and the distance $X_{\mathrm{GSM}}$ at the apex of the magnetic field line (down-up approach).

We then examine auroral motions occurring during growth phases of substorms and address the question: is the equatorward motion of the auroral arc well reproduced by the T01 model?

\subsection{Comparison between SI12 observations and T01 model during very quiet periods}

Sergeev's numerical simulations (Sergeev et al., 1983) indicate that the IB corresponds to $R=8$ for particles scattered to the center of the loss cone. The maximum of the precipitation is where the loss cone is completely filled, located where $R=6$. However, all these simulations were performed for $80 \mathrm{keV}$ protons, while SI12 mostly responds to protons between $\sim 2$ and $\sim 20 \mathrm{keV}$. The choice of high proton energies in the Sergeev's studies was pragmatic since they preferred to simulate energies high enough so that the flux is not significantly modified by field-aligned acceleration/deceleration. However, Sergeev et al. (1983) showed that these critical $R$ values are energy-independent. Therefore, since the aim of this study is to compare the T01 model with SI12 data, we always calculate the particle gyroradius $\rho$ and the corresponding $R_{c} / \rho$ ratio for $10 \mathrm{keV}$ protons. Our assumption of $10 \mathrm{keV}$ protons is also justified by the value of the plasmasheet temperature near the peak location which is around $10 \mathrm{keV}$ (within a factor 2 for over $90 \%$ of the observations). The location of the calculated MAX and IB for a $10 \mathrm{keV}$ 

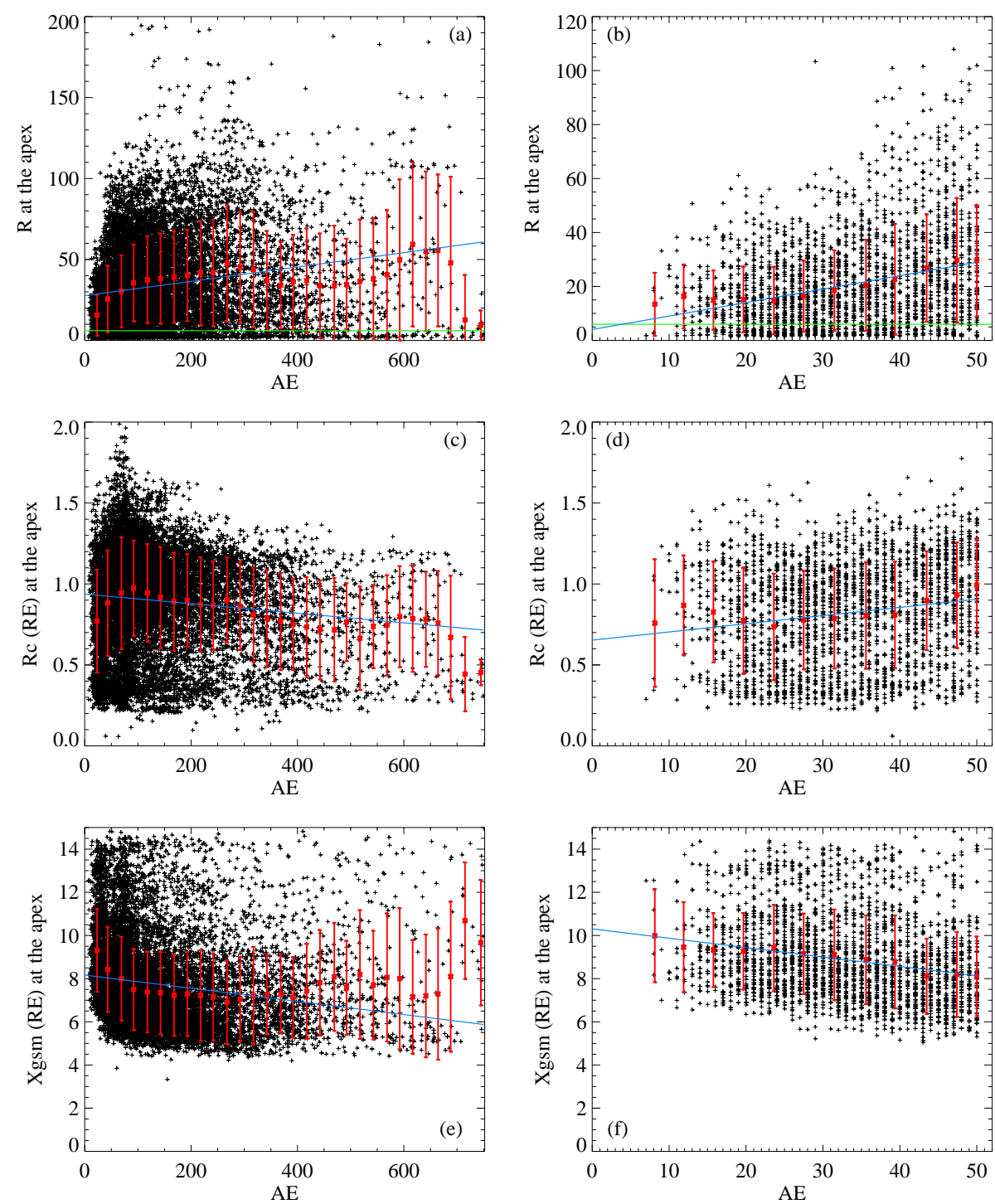

Fig. 3. Distribution of the $R$ value $(\mathbf{a}, \mathbf{b})$, the curvature radius $R_{C}(\mathbf{c}, \mathbf{d})$, and the distance $X_{\mathrm{GSM}}(\mathbf{e}, \mathbf{f})$ at the apex of the magnetic field line determined from MAX-SI12 using T01 as a function of the AE auroral activity index. The data base includes 17874 SI12 images collected in December 2000 and January 2001. The left plots (a, c, e) show all cases, and the right plots (b, d, f) AE smaller than 50. The red points (with standard deviation bars) represent the mean value over a fixed interval of AE, the green line is the expected value $R=6$ at the maximum of the proton precipitation, and the blue line is the linear regression over the entire data set.

proton energy is thus expected to best matched to the SI12 observations.

As the SI12 observations does not directly provide the position of the IB (we can obtain it with a statistical calculation), we rather compare MAX-SI12 with the latitude of the footprint of the T01 magnetic field line satisfying the condition $R=6$ (the MAX calculated from T01). The footprint of the T01 magnetic field line which satisfies $R=8$ (the IB calculated from T01) is sometimes showed as an example.

In this approach, this comparison rarely shows a good agreement between the latitude of MAX-SI12 in the ionosphere and that of the MAX (and IB) calculated from the T01 model at 00:00 MLT. Indeed, the calculated MAX is generally found several degrees poleward of the observed maximum of the proton precipitation. Figure 1 shows as an example a $4 \mathrm{~h}$ extremely quiet period with a positive $B_{z}$, on 5 November 2000, between 04:30 UT and 08:30 UT. In this case, as in the most of quiet cases, the calculated MAX (in blue), as well as the calculated IB (in black), is located 2-3 degrees poleward of MAX-SI12 (in red). This result implies that, either the T01 model underestimates the stretching of the magnetic field, or the IB location is controlled by a ratio $R=R c / \rho$ less than 8 . 


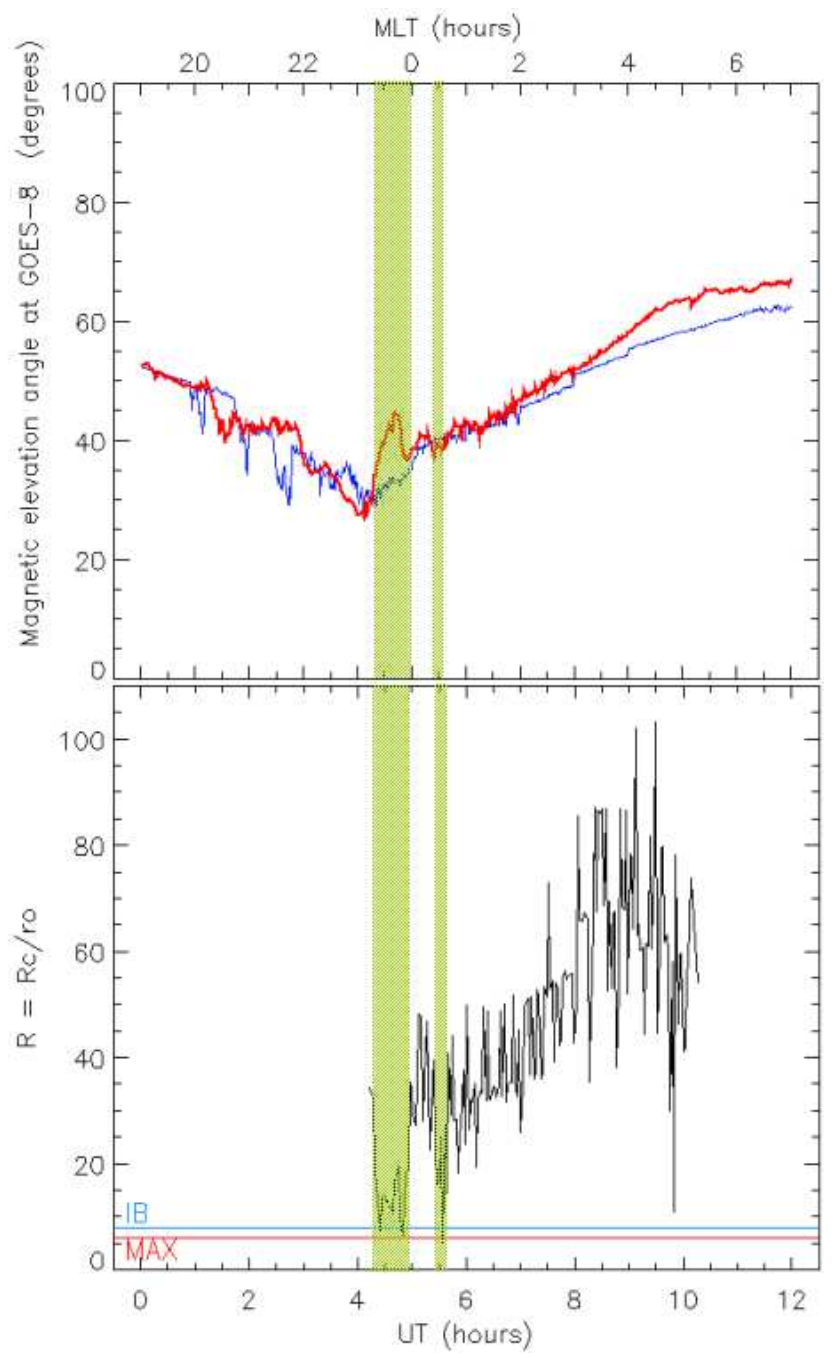

Fig. 4. The top panel shows an example of a 12-h period on 5 November 2000, where the magnetic elevation angle calculated by T01 at the GOES-8 position (blue line) is close to the GOES-8 measurements (red line). The panel below shows the $R$ value calculated from the position of MAX-SI12 in the midnight sector at the same time. The grey areas show the regions where $R$ is close to the expected value ( $R=6$ for the maximum, showed by the red line).

Figure 2 shows the T01 magnetic field lines calculated at 06:30 UT on 5 November 2000 (during the quiet period shown previously). Their footprints coincide with the latitude of MAX-SI12 at the same time (64.3 ${ }^{\circ}$ MLAT). The thin line is the output of the unmodified T01 model, which gives the value of the $R_{c} / \rho$ ratio of 40.2 at the apex (at the diamond). The current sheet half-thickness $D$ is assumed to be a constant parameter in the T01 model and its best-fit value derived from the entire body of data is equal to $2.34 R_{E}$. The thick line is the result of the model where we have adjusted the thickness of the model current sheet to match the expected value of $R_{C} / \rho=6$ for the maximum of the proton precipitation at the apex. In this case, to force a sufficiently stretched magnetic configuration during this extremely quiet period, the current sheet half-thickness $D$ has been set equal to $0.69 R_{E}$.

The second method is numerically faster and allows to process a large number of images. Figures $3 a$ and $b$ show how the $R$ values deduced from the location of MAX-SI12 using T01 are distributed as a function of the auroral activity (measured by the AE index). The red points (with standard deviation bars) represent the mean value over a fixed interval of $\mathrm{AE}$, the green line is the critical value $R=6$ at the maximum of the proton precipitation, and the blue line is the linear regression over the entire data set. For small values of $\mathrm{AE}$ ( $\mathrm{AE}<50$, i.e. quiet conditions) (Fig. 3b), $R$ increases with activity. Similarly, $R_{C}$ at the apex of the field line becomes larger when $\mathrm{AE}$ increases (Fig. 3d) and the apex of the MAX-SI12 magnetic field line moves closer to the Earth (Fig. 3e), so the field becomes more dipolar. Indeed, during such conditions (very small AEs) the magnetic field lines appear to be stretched to the same extent as when $\mathrm{AE}$ is larger than 500. For more disturbed periods (AE $>100)$ (Fig. 3a), the $R c / \rho$ ratio remains stable, whereas $R_{C}$ slightly decreases as the field configuration stretches (Fig. 3c), while the apex does not really move tailwards except when AE reaches very high value (more than 400) (Fig. 3e). If we consider that $\mathrm{AE}<50$ represent mostly quiet periods, the mean value of $R$ deduced from our mapping of the SI12 maximum precipitation during quiet periods is between $\sim 10$ (for smallest $\mathrm{AE}$ indices) and $\sim 30$ (for $\mathrm{AE} \sim 50$ ), and can reach $\sim 50$ for largest AEs. This implies again that the T01 model does not produce enough stretching of the field lines, even during quiet cases.

A large scatter of $R$ values is observed about the mean for any energy interval. Part of this scatter is linked to inaccuracies in the determination of the location of the peak proton brightness, and probably to the diversity of particular situations such as substorms or shock-induced aurora which are present in the statistics, but other effects are clearly present. A large contribution probably stems from the inadequacy of the Tsyganenko model to represent the actual field line geometry in the vicinity of the plasma sheet for a given specific time. Such models cannot reproduce the variability arising due to the internal dynamics, e.g. substorm phase). A considerable fraction of this scatter can be explained/reduced by monitoring the concurrent magnetic field in the magnetosphere as was shown by Ganushkina et al. (2005).

Coming back to the same quiet period on 5 November 2000, the top panel of Fig. 4 shows the T01 model is in a good agreement with the GOES-8 measurements of the field inclination angle during at least a few hours, which is a rare occurrence. The red line is the magnetic elevation angle as measured by GOES- 8 during a geostationary orbit, while the blue line represent the calculated (T01 used only) magnetic elevation angle at the GOES-8 position. The bottom panel shows the $R$ value calculated from the position of MAX-SI12 in the midnight sector at the same time. The green areas show the regions where $R$ is close to the expected value (i.e. 6 for 
the maximum). It is seen that $R$ is generally overestimated, as in many other comparisons, but in this case it remains reasonably close to the measurements. However, the best agreement between the T01 model and the in situ measurements is not exactly coincident with conditions where $R$ is closest to the theoretical value. The T01 model rarely provides a magnetospheric configuration where the calculated magnetic elevation angle is close (within 3-4 degrees) to that observed by GOES-8 during more than a few minutes. Moreover, even when the T01 inclination angle is quite close to the measurements such as this example between 00:00 and 08:00 UT, the Sergeev criterion may be mistaken by a factor of 2 or 3 . This suggests that, even though the actual field is sometimes well reproduced by the T01 model at the geostationary orbit, it is insufficiently stretched at the apex distance (as shown in Fig. 5). Therefore Pulkkinen's (1991) conclusion that the magnetic field is in a more tail-like configuration than predicted by the T89 model during growth phases also applies to the T01 model, including during non growth phase quiet periods.

We conclude that the T01 model is inadequate to predict the actual geometry of the stretched field lines outside geostationary orbit in the vicinity of the plasma sheet. One thus cannot expect to find the calculated IB location to be close to the observed SI12 maximum. The actual field lines are more stretched than those predicted by T01, so that the $R$ value calculated with the model will generally exceed the actual value associated with the isotropy boundary.

\subsection{Growth phases}

The growth phase is an interesting period to study the behavior of the magnetic field lines in the tail of the magnetosphere. Indeed, during the $30 \mathrm{~min}$ or more preceding the substorm onset, the magnetic field slowly stretches, and the degree and rate of the stretching can be easily correlated with the solar wind conditions and other geomagnetic indices. The stretching also produces an equatorward motion of the footprint of the magnetic field lines, which can be observed with a global imager such as SI12 on board IMAGE. Figure 6 shows an example of a growth phase observed on 26 August 2000. Figure 6a is a keogram of the proton precipitation located observed with SI12 in the midnight sector (between 23:30 and 00:30 MLT). Figure 6b is the keogram in the onset sector (between 21:30 and 22:30 MLT). During the growth phase preceding the substorm onset, a well marked equatorward motion of the precipitation is observed until about 09:30 UT when the substorm onset appears in the 22:00 MLT sector. The equatorward motion extends further in time, until 09:50 UT in the midnight sector.

In Fig. 7, we show the equatorial (triangles) and polar (pluses) boundaries in the one-hour wide sector around midnight during the same event as before. These boundaries are found were the SI12 signal falls to zero, following removal of the background counts. The diamonds represent the max-

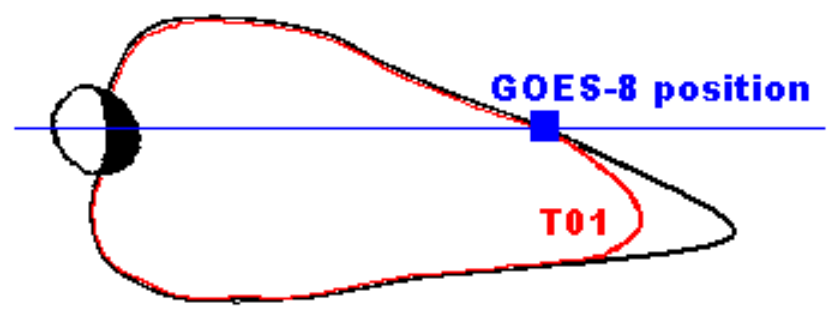

Fig. 5. Sketch of a T01 magnetic field line (red line) which is not stretched enough and has a too large radius of curvature value at the apex, while the magnetic elevation angle at the GOES-8 position (blue square) is close to the actual configuration (black line).

imum of the proton precipitation, and the dotted line is the value of the calculated IB for the solar wind conditions and ground disturbance at this event. Both boundaries and the SI12 maximum have an unequivocal equatorward motion (by more than $3^{\circ}$ MLAT), whereas the calculated IB does not show any clear motion during the growth phase of this substorm. This behavior is typical of the 26 growth phases analyzed between May 2000 and December 2001. These events have been selected because they show a strong equatorward motion of the equatorial and polar boundaries, but the calculated IB almost never exhibits significant latitudinal motion. Moreover, the IB calculated from the T01 model and the Sergeev's criterion is typically found close to the polar side of the maximum. This result also confirms the studies of Pulkkinen (1991) and Pulkkinen et al. (1992) indicating that any equatorward motion of the magnetic field lines during the growth phase, modeled with the T01 as well as the T89 models, requires an increase of the near-Earth currents to progressively adjust toward a more tail-like configuration. The resulting stretching is already underestimated one hour before the onset, as we previously noted for non growth phase quiet periods.

\section{Discussion}

Recent studies have shown that a direct relation exists between the maximum proton precipitation observed with a remote sensing imager such as SI12 and the magnetic elevation angle (Blockx et al., 2005). Indeed, the location of the SI12 maximum intensity (MAX-SI12) was shown to be, as well as the isotropy boundary (IB) determined from in situ measurements, a good indicator of the stretching of the magnetic field lines. Blockx et al. (2005) demonstrated that both the IB and MAX-SI12, unlike the usual global activity indices (magnetic indices like $\mathrm{AE}, K_{p}, D_{s t}$ ), are well suited to predict the instantaneous magnetospheric configuration in the midnight sector. Taking into account these results, we have compared the SI12 observations with the results of the T01 empirical model of the magnetospheric tail structure. An earlier version of the Tsyganenko model (T89) was already 


\section{FUV Keogram/SI12 28 Aug 2000}
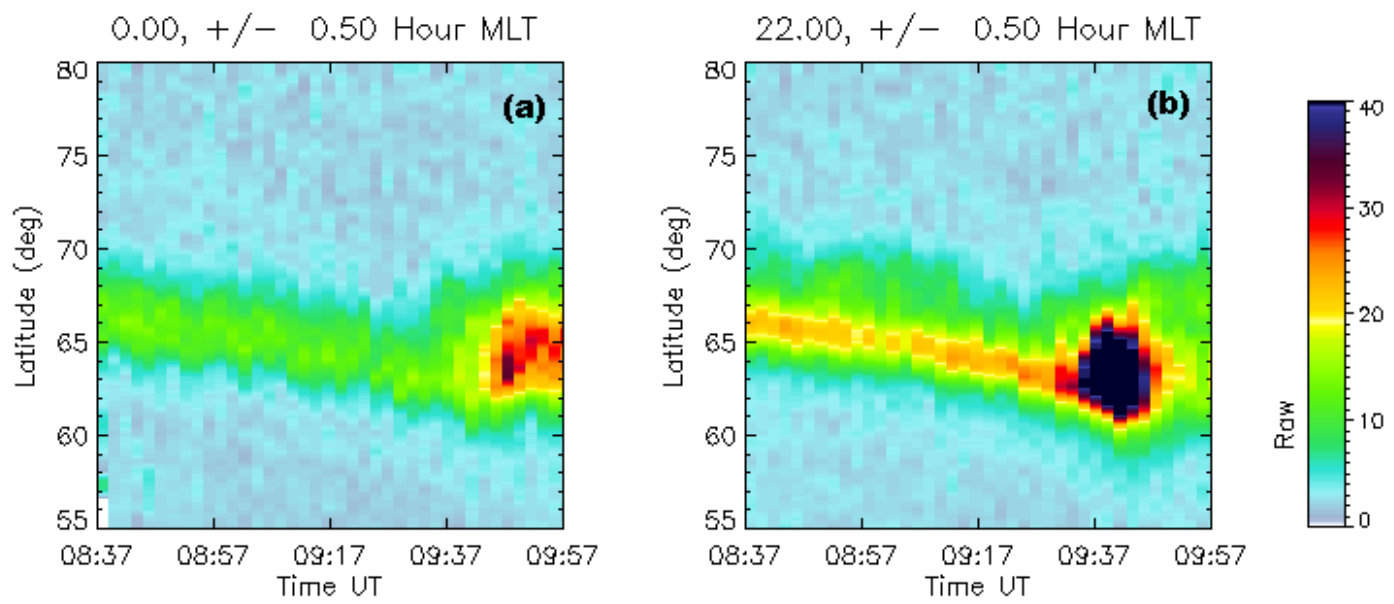

Fig. 6. Example of a growth phase observed on 28 August 2000 event. Panel (a) is a keogram of the proton precipitation observed with SI12 in the midnight sector (between 23:30 and 00:30 MLT). Panel (b) is the keogram in the onset sector (between 21:30 and 22:30 MLT in this case).

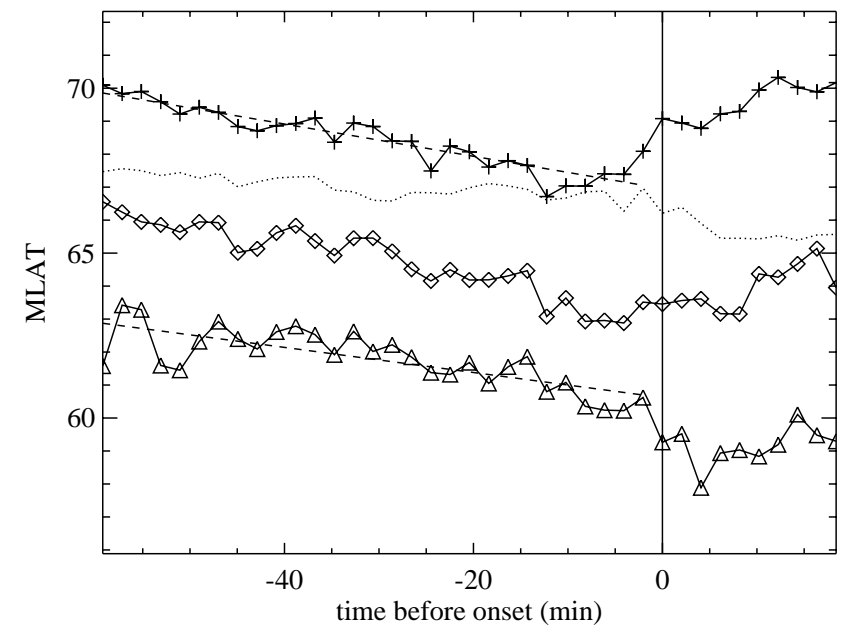

Fig. 7. Evolution of the equatorial (triangles) and polar (pluses) boundaries in the one-hour wide sector around midnight during the same event as before. The diamonds represent the maximum of the proton precipitation, and the dotted line is the value of the calculated IB for the solar wind conditions and $D_{s t}$.

confronted with the location of auroral arcs recorded by allsky cameras or with the magnetic field $\mathrm{B}_{x}$ and $\mathrm{B}_{z}$ components observed by GOES-5 by Pulkkinen (1991) and Pulkkinen et al. (1992). They showed that the geometry of the T89 magnetic field lines is not enough tail-like, in particular during the growth phase of a substorm event. They found, by studying several growth phase events, that the observed configuration may be reproduced by enhancing the near-Earth currents and thinning the current sheet from the values given by the static T89 model. In this work, we focused on the 2001 version of the Tsyganenko model (T01) and its comparison with global view of auroral proton precipitation provided by the SI12 images.

The comparison with the location of MAX-SI12 showed that the T01 model underestimates the stretching and is not enough tail-like, as was found for T89. This conclusion applies to growth phases, and during quiet conditions as well. The latitude of the magnetic field line footprint of the line which meets the Sergeev's criterion ( $R=6$ for the maximum proton precipitation) is generally higher than the latitude of the observed MAX-SI12. However, we showed that it is possible to match the observed location of MAX-SI12 by forcing the model using a decreased current sheet half-thickness $D$ parameter, down to $0.69 R_{E}$ in the case illustrated, instead the default value of $2.34 R_{E}$. With this smaller current sheet thickness, the geometry of the magnetic field lines becomes more stretched (more tail-like), as was found in a quite similar approach by Pulkkinen (1991), and Kubyshkina et al. (1999) for the static T89 model. Indeed, Kubyshkina et al. (1999) found a minimal current sheet half-thickness $D$ between 0.1 and $0.7 R_{E}$ just before substorm onset for several events. The study of growth phase events confirms that using T01 during pre-onset periods provides adequate results only by adding a sheet current to the model or changing parameters such as the current sheet half-thickness. Indeed, the principal reason of the discrepancy between the observed IB positions and the corresponding model values of the $R$ parameter is the excessively large value of the current sheet half-thickness $D$ in the T01 model, equal to $2.34 R_{E}$ and assumed independent of the magnetospheric state. The resultant large systematic overestimate of $R$, clearly seen 

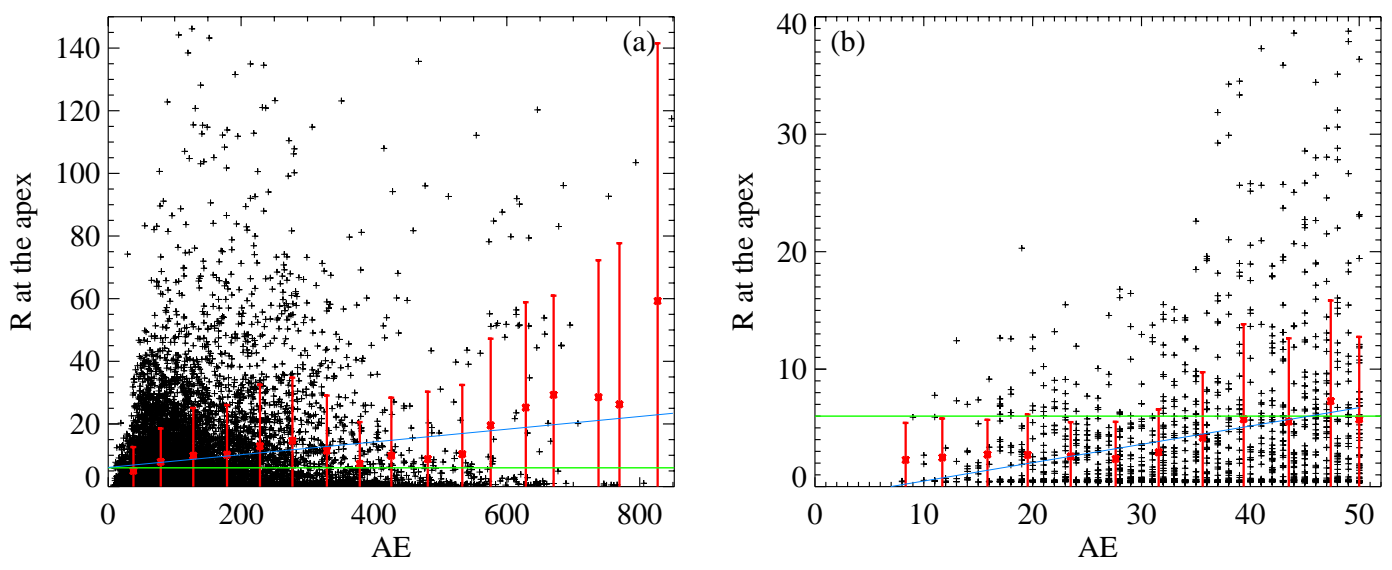

Fig. 8. Same plot as Figs. 3a, b, except the $R$ value has been obtained using a smaller tentative fixed value of the current sheet half-thickness $D\left(0.5 R_{E}\right)$.

in Figs. 3a, b, can be eliminated by setting a significantly smaller half-thickness: Figs. 8a, b is a modified version of the two top panels (a, b) of Fig. 3, obtained using a smaller tentative fixed value of $D\left(0.5 R_{E}\right)$. It definitively results in much a better agreement between the blue (model value of $R$ ) and green (theoretical value) lines. However, such a correction cannot remove the residual noise, because of the largely unpredictable nature of the deviations in each individual case since the T01 model does not consistently predict the same discrepancy with observation. This noise, as well as the relatively large value of $D$ derived in the T01 model from data, is a natural consequence of the highly unstable and variable field in the near plasma sheet (e.g. Borovsky et al., 2003) and a large variety of possible scenarios in the dynamics of the inner magnetosphere. As demonstrated by Sergeev (2003) on the basis of Cluster data, the magnetic structure of the tail plasma sheet is indeed highly dynamical and turbulent, which is far from the idealized model with a regular and smooth current sheet with a fixed thickness. The individual irregular variations of the magnetic field are very unlikely to be faithfully reproduced by any empirical model with a simple external input, such as in the T01 model. This viewpoint has been developed earlier by Kubyshkina et al. (1999) and it is at the core of their "event-oriented" approach.

Acknowledgements. J.-C. Gérard and B. Hubert are supported by the Belgian National Fund for Scientific Research (FNRS). The IMAGE-FUV investigation was supported by NASA through SWRI subcontract 83820 at the University of California, Berkeley, contract NAS5-96020. This work was funded by the PRODEX program of the European Space Agency (ESA) and the Fund for Collective and Fundamental Research (FRFC grant \#2.4517.02). ACE level 2 data were provided by N. F. Ness (MFI) and D. J. McComas (SWEPAM), and the ACE Science Centre. GOES-8 data were obtained thanks to CDAWeb. $\mathrm{AE}$ and $D_{s t}$ indices are provided by the World Data Center for Geomagnetism (WDC-C2) in Kyoto. We thank the reviewers for their constructive comments on the manuscript.
Topical Editor I. A. Daglis thanks T. Pulkkinen and N. Tsyganenko for their help in evaluating this paper.

\section{References}

Bisikalo, D. V., Shematovich, V. I., Gérard, J.-C., Meurant, M., Mende, S. B., and Frey, H. U.: Remote sensing of the proton aurora characteristics from IMAGE-FUV, Ann. Geophys., 21, 2165-2173, 2003, http://www.ann-geophys.net/21/2165/2003/.

Blockx, C., Gérard, J.-C., Meurant, M., Hubert, B., and Coumans, V.: Far ultraviolet remote sensing of the isotropy boundary and magnetotail stretching, J. Geophys. Res., 110, A11215, doi:10.1029/2005JA011103, 2005.

Borovsky, J. E. and Funsten, H. O.: MHD turbulence in the Earth's plasma sheet: Dynamics, dissipation, and driving, J. Geophys. Res, 108(A7), 1284, doi:10.1029/2002JA009625, 2003.

Coumans, V., Gérard; J.-C., Hubert, B., and Evans, D. S.: Electron and proton excitation of the FUV aurora: simultaneous IMAGE and NOAA observations, J. Geophys. Res., 107(A11), 1347, doi:10.1029/2001JA009233, 2002.

Coumans, V., Gérard, J.-C., Hubert, B., Meurant, M., and Mende, S. B.: Global auroral conductance distribution due to electron and proton precipitation from IMAGE-FUV observations, Ann. Geophys., 22, 1595-1611, 2004, http://www.ann-geophys.net/22/1595/2004/.

Donovan, E. F., Jackel, B. J., Voronkov, I., Sotirelis, T., Creutzberg, F., and Nicholson, N. A.: Ground-based optical determination of the b2i boundary: A basis for an optical MT-index, J. Geophys. Res., 108, 1115, doi:10.1029/2001JA009198, 2003.

Frey H. U., Mende, S. B., Carlson, C. W., Gérard, J. C., Hubert, B., Spann, J., Gladstone, R. G., and Immel, T. J.: The electron and proton aurora as seen by IMAGE-FUV and FAST, Geophys. Res. Lett., 28, 1135-1138, 2001.

Frey, H. U., Mende, S. B., Immel, T. J., Gérard, J. C., Hubert, B., Habraken, S., Spann, J., Gladstone, G. R., Bisikalo, D. V., and Shematovich, V. I.: Summary of quantitative interpretation of IMAGE far ultraviolet auroral data, Space Sci. Rev., 109, 255283, 2003. 
Ganushkina, N. Yu., Pulkkinen, T. I., Kubyshkina, M. V., Sergeev, V. A., Lvova, E. A., Yahnina, T. A., Yahnin, A. G., and Fritz, T.: Proton isotropy boundaries as measured on mid- and low-altitude satellites, Ann. Geophys., 23, 1839-1847, 2005, http://www.ann-geophys.net/23/1839/2005/.

Gérard, J. C., Hubert, B., Bisikalo, D. V., and Shematovich, V. I.: A model of the Lyman $\alpha$ line profile in the proton aurora, J. Geophys. Res., 105, 15 795-15 805, 2000.

Gérard, J.-C., Hubert, B., Meurant, M., Shematovich, V. I., Bisikalo, D. V., Frey, H. U., Mende, S. B., Gladstone, G. R., and Carlson, C. W.: Observation of the proton aurora with IMAGE FUV imager and simultaneous ion flux in situ measurements, J. Geophys. Res., 106, 28 939-28 948, 2001.

Hubert, B., Gérard, J.-C., Evans, D. S., Meurant, M., Mende, S. B., Frey, H. U., and Immel, T. J.: Total electron and proton energy input during auroral substorms: Remote sensing with IMAGEFUV, J. Geophys. Res., 107, 1183, doi:10.1029/2001JA009229, 2002.

Kubyshkina, M. V., Sergeev, V. A., and Pulkkinen, T. I.: Hybrid Input Algorithm: An event-oriented magnetospheric model, J. Geophys. Res., 104(A11), 24 977-24 993, 1999.

Mende, S. B., Heetderks, H., Frey, H. U., Lampton, M., Geller, S. P., Habraken, S., Renotte, E., Jamar, C., Rochus, P., Spann, J., Fuselier, S. A., Gérard, J.-C., Gladstone, R., Murphree, S., and Cogger, L.: Far ultraviolet imaging from the IMAGE spacecraft. 1. System design, Space Sci. Rev., 91, 243-270, 2000a.

Mende, S. B., Heetderks, H., Frey, H. U., Lampton, M., Geller, S. P., Abiad, R., Siegmund, W., Tremsin, A. S., Spann, J., Dougani, H., Fuselier, S. A., Magoncelli, A. L., Bumala, M. B., Murphree, S., and Trondsen, T.: Far ultraviolet imaging from the IMAGE spacecraft. 2. Wideband FUV imaging, Space Sci. Rev., 91, 271285, 2000b.

Meurant, M., Gérard, J.-C., Hubert, B., Coumans, V., Shematovich, V. I., Bisikalo, D. V., Evans, D. S., Gladstone, G. R., and Mende, S. B.: Characteristation and dynamics of the auroral electron precipitation during substorms deduced from IMAGE-FUV, J. Geophys. Res., 108(A6), 1247, doi:10.1029/2002JA009685, 2003.

Newell, P. T., Sergeev, V. A., Bikkuzina, G. R., and Wing, S.: Characterizing the state of the magnetosphere : Testing the ion precipitation maxima latitude (b2i) and the ion isotropy boundary, J. Geophys. Res., 103, 4739-4745, 1998.

Nicholson, N. A., Donovan, E. F., Jackel, B. J., Voronkov, I., Cogger, L. L., Lummerzheim, D., Creutzberg, F., and Sotirelis, T.: Using the optical b2i as a tool for event timing, paper presented at the 6th International Conference on Substorms (ICS-6), NASA, Seattle, Wash., 25-29 March 2002.

Pulkkinen, T. I.: A study of magnetic field and current configurations in the magnetotail at the time of a substorm onset, Planet. Space Sci., 39, 833-845, 1991.
Pulkkinen, T. I., Baker, D. N., Pellinen, R. J., Büchner, J., Koskinen, H. E., Lopez, R. E., Dyson, R. L., and Frank, L.A.: Particle scattering and current sheet stability in the geomagnetic tail during the substorm growth phase, J. Geophys. Res., 97(A12), 19283 $19297,1992$.

Sergeev, V. A. and Gvozdevsky, B. B.: MT-index - a possible new index to characterize the magnetic configuration of magnetotail, Ann. Geophys., 13, 1093-1103, 1995, http://www.ann-geophys.net/13/1093/1995/.

Sergeev, V. A., Sazhina, E. M., and Tsyganenko, N. A.: Pitch-angle scattering of energetic protons in the magnetotail current sheet as the dominant source of their isotropic precipitation into the nightside ionosphere, Planet. Space Sci., 31, 1147-1155, 1983.

Sergeev, V. A., Malkov, M. V., and Mursula, K.: Testing of the isotropic boundary algorithm method to evaluate the magnetic field con®guration in the tail, J. Geophys. Res., 98, 7609-7620, 1993.

Sergeev, V. A., Runov, A., Baumjohann, W., Nakamura, R., Zhang, T. L., Volwerk, M., Balogh A., Reme H., Sauvaud, J. A., Andre, M., and Klecker, B.: Current sheet flapping motion and structure observed by Cluster, Geophys. Res. Lett., 30(6), 1327, doi:10.1029/2002GL016500, 2003.

Strickland, D. J., Daniell, R. E., Jasperse, J. R., and Basu, B.: Transport-theoretic model for the electron-proton-hydrogen atom aurora, 2, Model results, J. Geophys. Res., 98, 21533 $21548,1993$.

Tsyganenko, N. A. and Usmanov, A. V.: Determination of the magnetospheric current system parameters and development of experimental geomagnetic field models based on data from IMP and HEOS satellites, Planet. Space Sci., 30, 985-998, 1982.

Tsyganenko, N. A.: Global quantitative models of the geomagnetic field in the cislunar magnetosphere for different disturbance levels, Planet. Space Sci., 35, 1347-1358, 1987.

Tsyganenko, N. A.: A magnetospheric magnetic field model with a warped tail current sheet, Planet. Space Sci., 37, 5-20, 1989.

Tsyganenko, N. A.: Modelind the Earth's magnetospheric magnetic field confined within a realistic magnetopause, J. Geophys. Res., 100(A4), 5599-5612, 1995.

Tsyganenko, N. A.: A model of the near magnetosphere with a dawn-dusk asymmetry: 1. Mathematical structure, J. Geophys. Res., 107(A8), 1179, doi:10.1029/2001JA000219, 2002a.

Tsyganenko, N. A.: A model of the near magnetosphere with a dawn-dusk asymmetry: 2. Parameterization and fitting to observations, J. Geophys. Res., 107(A8), 1176, doi:10.1029/2001JA000220, 2002b. 